\title{
Cooperation of Knowledge Based Systems for Galaxy Classification
}

\author{
Jean-Christophe Ossola and Monique Thonnat \\ INRIA, Sophia Antipolis BP 93, France
}

\begin{abstract}
Astronomical observing systems provide astronomers with a great amount of high quality data. Thus, there is a need for automating data processing. In this paper, we are interested in the galaxy classification problem which is important for universe understanding. The classification consists of two main steps: parameter extraction for object description and data interpretation for object recognition. We present a cooperative architecture based on two knowledge-based systems for automatic classification. The first system is specialized in computer vision and allows parameter extraction. The second is dedicated to galaxy classification. This paper shows our methods and the main components of both knowledge-based system mentioned above.
\end{abstract}

\section{Introduction}

This paper presents a system for automatic classification of galaxies based on their images. Most of the current work in data interpretation by computer vision concerns the recognition of man-made objects [4] [5], and takes into account the three-dimensional aspect of objects [6]. In contrast, galaxies are natural complex objects which have no defined geometric models.

Recognizing complex natural objects, requires the cooperation of computer vision techniques, for image description, and data interpretation techniques for object, recognition. Artificial Intelligence, with knowledge-based systems (KBS) or neural network [8] may be useful to achieve this task. Neural networks do not reflect knowledge of experts contrary to KBS. Furthermore, KBS can evolve, are flexible and allow knowledge exploitation.

Automation of object classification requires a great amount of knowledge in both computer vision and data interpretation. As this cannot be handled by a single expert, we use a distributed approach to separate different sources of knowledge. Our distributed approach is based on two knowledge-based systems. A schematic view of the global architecture of the system is shown in figure 1 . The first stage computes, from the image, numerical parameters describing the galaxy morphology. This phase is performed by PROGAL [2], a knowledge-based system specialized in image processing. The second phase classes the galaxy described by the numerical parameters computed in the first phase, using a taxonomy closed to de Vaucouleurs classification [3]. This phase provides as output the morphological type plus a detailed symbolic description of the galaxy. It is performed by SYGAL [9], a knowledge-based system dedicated to galaxy classification. In the next sections, we will describe PROGAL and SYGAL. Section 


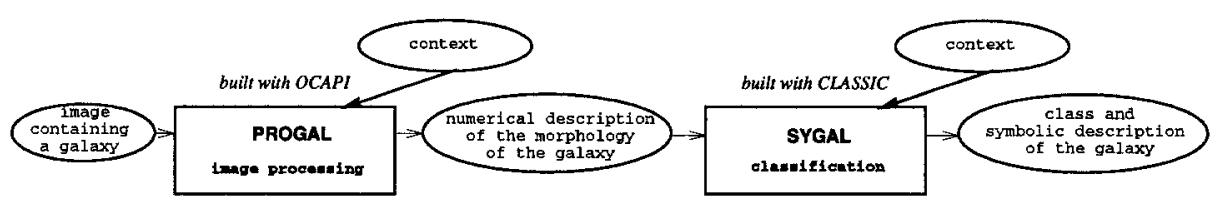

Fig. 1. Synopsis of the global processing of images containing a galaxy

4 gives an overview of the communication protocol and section 5 shows some results. Finally, we conclude with remarks on future works.

\section{PROGAL}

PROGAL is a KBS built with OCAPI [1], a KBS shell dedicated to program supervision. This means that, given a goal to achieve and a library of programs, pertinent programs are chosen, ordered and their execution is controlled. The PROGAL knowledge base contains several types of useful knowledge described below. A program description is associated to each image processing program in the library. It is a description of the program function, input data, output data, tunable input parameters and calling syntax. An important kind of knowledge is also predefined typical decompositions of image processing goals into several sequential or parallel sub steps. Rules are explicit descriptions of conditions under which certain decisions are to be taken. Four different types of rules are used. Choice rules explicit the way the selection is operated among several programs or sequences of treatment to reach a goal. Initialization rules explicit the method used to compute the value of the input parameters of a selected program. Evaluation rules explicit how the output data of the programs are assessed. Adjustment rules explicit how to modify the value of parameters after a negative evaluation of the results of a program.

The global processing, named Morphological-description is described in a typical decomposition into five sub-steps:

(1) Creation and initialization of the file containing the numerical parameters,

(2) Extraction in the image of the object of interest, (3) Computing of global parameters describing the galaxy, (4) Building of 5 iso-intensity contours corresponding to different regions of the galaxy, and (5) For each of these contours, computation of numerical parameters describing them.

Among these steps, two are relatively complex and are decomposed in substeps. Extraction of the galaxy is performed as follows: (1) localization of the center of the galaxy, (2) effective isolation of the galaxy and (3) noise removal. Building of iso-intensity contours is decomposed into: (1) Creation of thresholded images and (2) Extraction of the contours which are significant.

The knowledge base of PROGAL contains 34 program descriptions, 25 typical decompositions, 12 choice rules, 6 initialization rules, 5 evaluation rules, and 4 adjustment rules. 


\section{SYGAL}

We used CLASSIC [10], a KBS shell, to develop the SYGAL knowledge based system in order to perform classification in the same way experts do, using their usual terminology. CLASSIC facilitates additions of new classes as well as it enables the completion of galaxy descriptions. Models of known object classes are described by prototypes which are structured objects organized in a tree reflecting the specialization relations between the different object classes. Prototypes are described in terms of descriptors with symbolic or numerical values. Inference rules, organized in rule bases attached to prototypes enable data abstraction. Their role is to allow the passage from numerical input parameters to symbolic values assigned to prototype descriptors. A fuzzy comparison mechanism allows to handle noisy, incomplete or imprecise data. To classify an object means to find the closest corresponding prototype or prototypes and to give a correspondence measure.

The inference engine works as follows: At each node of the prototype tree, rules attached to this prototype are activated, the object to be classified is matched against the current prototype. If a prototype is validated, the system selects its sons, if not, the search stops. The SYGAL knowledge base contains 117 rules and 41 prototypes. Examples of a prototype and two rules are shown in the table below:

\begin{tabular}{|c|c|c|}
\hline Prototype SA3 & & \\
\hline profile: $[0,10000]$ & ellipticity: $[-.9,1]$ & orientation: $[-90,90]$ \\
\hline linear-error: $[0,100000]$ & area: $[0,900000]$ & shape: spiral \\
\hline $\begin{array}{c}\text { isophotes: smooth, normal } \\
\text { distorted }\end{array}$ & $\begin{array}{c}\text { centering: good, average } \\
\text { mediocre }\end{array}$ & resolution: good \\
\hline bar: absent & arms: incipient & \\
\hline c1: contour & c2: contour & c3: contour \\
\hline c4: contour & c5: contour & \\
\hline RULE 95 & RULE 59 & \\
\hline $\begin{array}{l}\text { Class: contour } \\
\text { if eccentricity }<0.65 \\
\text { then shape = average }\end{array}$ & $\begin{array}{l}\text { Class: galaxy } \\
\text { if resolution }=\text { good } \\
\text { then bulge }=\text { visible }\end{array}$ & and linear-error $>0.02$ \\
\hline
\end{tabular}

\section{The communication protocol}

In order to make PROGAL and SYGAL cooperate, we use a communication language called $\mathrm{CHOOE}$ [7]. It allows to create communication networks between such systems. Communication is made by message sending in different modes (synchronous, asynchronous and deferred). We mostly use the deferred mode: a system waiting for an answer to a message it has sent is still able to answer another message. There is no conflict to manage because each system is highly specialized for a task (image processing or classification) and manages its own control.

An example of message exchanged between PROGAL and SYGAL is shown below: 


\begin{tabular}{|l|ll|}
\hline Name & Classification-request-1 & \\
\hline Communication & mode: deferred & service: object-classification \\
\hline Content & input-data: NGC7531t.results \\
\hline Context & noise: absent & stars: absent \\
& image-type: density & galaxy-location: unknown \\
\hline
\end{tabular}

\section{Automatic classification}

This section presents some results obtained by the global system on several galaxies, some of which are shown in figure 2.

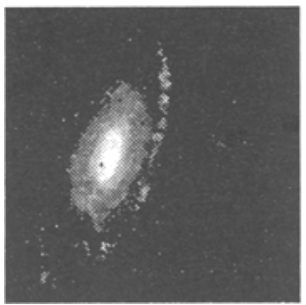

NGC 7531

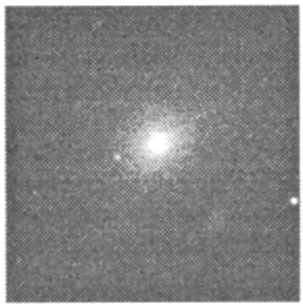

$N G C 4406$

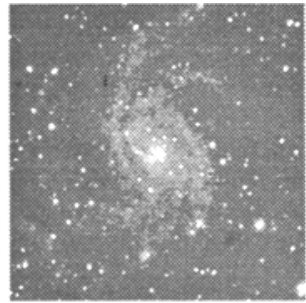

NGC 6936

Fig. 2. Some Galaxies

The external contextual information provided to PROGAL for these images are different. For example, the first image, galaxy location is unknown, noise is absent, stars are absent. For the second image, the galaxy is centered in the image, noise is present, and stars are present. The third galaxy is centered, noise is present, and stars are present. The classification of galaxies is performed as follows. Given an image of a galaxy, an external initial request is made on the image processing goal morphological-description. This request triggers the five previously mentioned sub-steps. (1) The extraction of the object of interest uses a choice rule to choose between two possible decompositions. If we have no a priori information on the position of the galaxy in the image, we look for the most important object present in the image. To check that we have actually detected a galaxy and not a star, an evaluation rule compares the size of the most important object with the size of the maximal star size in the observed region. If the detection is not correct an adjustment rule decreases the threshold values. (2) Then, we compute five global parameters describing the galaxy: Two photometric parameters computed using the projected profile along the main axis (see figure 3 ) and three geometrical parameters.

(4) The five contours are built (see figure 3). (5) For each contour, six numerical parameters are computed.

Finally, PROGAL provides a set of numerical parameters to SYGAL which begins the classification process. SYGAL first checks that the input parameters of each contour are compatible with the description of the prototype contour. Rules attached to the contour prototype are activated and infer new values. Then 


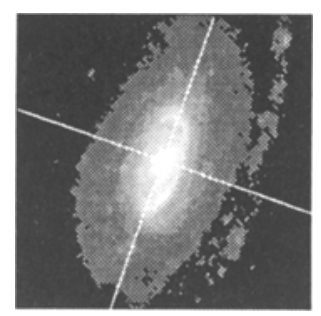

Extracted galaxy+axes

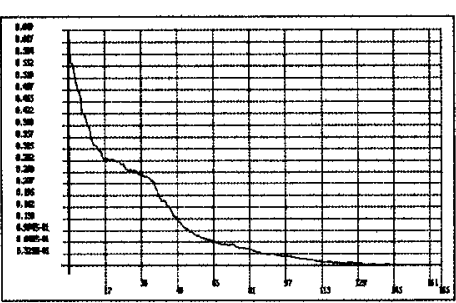

Projected profile (Main axis)

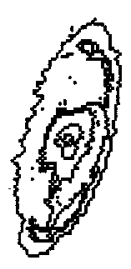

superimposed contours

Fig. 3. Photometric analysis (NGC7531)

for each of the five contours, the system validates one of the following prototypes: spiral, average, elliptical. When the five contours are classified, the galaxy prototype is validated. Once again, the system scans the rule base attached to the current prototype. Rules are activated, infer new values which allow the system to validate one or several prototypes among spiral, intermediate and early. This process continues until no more rule can be activated or a leaf of the prototype tree is reached.

We have tested this system on two sets of images containing respectively 11 and 8 images. For the first, images set were taken with a low resolution telescope (Schmitt), whereas they were taken with a high resolution one (MacDonald telescope) for the second set. Although the results are quite good, problems remain with estimation of arm size and bar detection. The knowledge base does not contain information about irregular galaxies and about ring pattern structures. Results are shown in the tables below.

\begin{tabular}{|c|c|c|c|c|}
\hline Name & Real class & Global result & Best result & Comment \\
\hline \multicolumn{5}{|c|}{ Schmitt telescope images } \\
\hline NGC 4421 & SBS0 & lb+ Sb1c & Sb1c & Almost correct \\
MGC 4540 & SA6 SB6 & SB3 SB5 & SB5 & Almost correct \\
NGC 4595 & SA3 SB3 & SA3 SB3 & SA3 SB3 & Correct \\
NGC 4474 & L & la+ lb+ Sa1c Sb1c & la+ lb+ & Correct \\
NGC 4569 & SA3 SB3 & SB5 SB7 & SB5 & To improve \\
NGC 4571 & SA7 & SA5 SA7 & SA5 & Correct \\
NGC 4639 & SA4 SB4 & SA5 SB5 & SA5 SB5 & Correct \\
NGC 4459 & la & la- & la- & Correct \\
NGC 4477 & lb & la- lb- la+ lb+ & la+ lb+ & Correct \\
NGC 4473 & E5 & E5 la+ lb+ & E5 & Correct \\
NGC 4406 & E3 & la+ & la + & To improve \\
\hline \hline \multicolumn{5}{|c|}{ MacDonald telescope images } \\
\hline NGC 1433 & SB5 & SB3 SA3 SB5 SA5 & SB3 SB5 & Correct \\
NGC 6300 & SB5 & SB5 & same & Correct \\
NGC 7531 & SA5 & SA5 & same & Correct \\
NGC 6946 & SA7 SB7 & SA7 SB7 & same & Correct \\
NGC 7702 & Sa1a & intermediate-b & same & To improve \\
\hline
\end{tabular}




\begin{tabular}{|c|c|c|c|c|}
\hline Name & Real class & Global result & Best result & Comment \\
\hline \multicolumn{5}{|c|}{ MacDonald telescope images } \\
\hline NGC 3021 & SA5 & SA5 & same & Correct \\
NGC 4027 & SB8 & SB5 & same & To improve \\
NGC 4303 & SA5 SB5 & SA & same & To improve \\
\hline
\end{tabular}

\section{Concluding remarks and future work}

We have presented our work on automatic galaxy classification by a distributed system. This system provides the necessary flexibility to process different kinds of images. For example, in [9], all images were taken with a low resolution telescope, and the image shown in section 5 has been taken with a high resolution one. The image processing required in the two cases is different, and our system is able to achieve it in both cases with respect to a certain context. In the future, the system needs to be extended to increase its capability of classification. Owing to our communication mechanism, sending specific requests on image processing programs is possible. For example, there is a crucial need for arm measurement and bar detection algorithms. We are currently working on it. A problem remains for translating quantitative descriptions issued from computer vision algorithms to qualitative description expressed in the data interpretation system. The use of a learning mechanism may provide help in this matter.

\section{References}

1. V. Clément and M. Thonnat. Integration of image processing procedures, OCAPI: a knowledge-based approach. Computer Vision Graphics and Image Processing : Image understanding, 57(2), March 1993.

2. V. Clément and M. Thonnat. Pilotage de procédures de traitement d'images pour la description morphologique de galaxies. TS, 9(5):389-401, January 1993.

3. G. de Vaucouleurs. Classification and morphology of external galaxies. Handbuch der Physik, 53:275, March 1957.

4. W. Eric and L. Grimson. Object Recognition by Computer : The role of Geometric Constraints. The MIT Press, 1990.

5. G. J. Ettinger. Large hierarchical object recognition using libraries of parameterized model sub-parts. In Computer Vision and Pattern Recognition, pages 32-41, 1988.

6. K. Ikeuchi and T. Kanade. Automatic generation of object recognition programs. IEEE, 76(8):1016-1035, August 1988.

7. F. Lebastard. CHOOE : un gestionnaire d'environnement distribué. Technical Report 93-22, CERMICS, December 1993.

8. M.C. Storrie-Lombardi, O. Lahav, L. Sodre, and L.J. Storrie-Lombardi. Morphological classification of galaxies by artificial neural networks. Mon. Not. R. Astronomical Society, 259:8p-12p, 1992.

9. M. Thonnat. The world of galaxies, chapter Toward an automatic classification of galaxies, pages 53-74. Springer-Verlag, 1989.

10. M. Thonnat and A. Bijaoui. Knowledge-Based Systems in Astronomy, chapter Knowledge-Based Classification of galaxies, pages 121-159. Springer-Verlag, 1989. 